\title{
Treatment of high-grade osteoblastic osteosarcoma of the humerus in a 5-year-old boy with cystic fibrosis: A case report
}

\author{
KENICHI V. OKUDA ${ }^{1}$, JUTTA HAMMERMANN ${ }^{1}$, BJÖRN S. LANGE ${ }^{1}$, \\ JANA C. FISCHER ${ }^{1}$, FALK THIELEMANN ${ }^{2}$, RALF KNÖFLER ${ }^{1}$ and MEINOLF SUTTORP ${ }^{1}$ \\ Departments of ${ }^{1}$ Pediatrics and ${ }^{2}$ Orthopedics and Traumatology, University Hospital \\ 'Carl Gustav Carus', Technical University, D-01307 Dresden, Germany
}

Received November 15, 2016; Accepted May 26, 2017

DOI: $10.3892 / \mathrm{mco} .2017 .1274$

\begin{abstract}
Antineoplastic treatment of osteoblastic osteosarcoma in a patient with cystic fibrosis (CF) may harbor a high risk of neutropenia-associated complications, and, to the best of our knowledge, has not been previously reported. Diagnosis of $\mathrm{CF}$ was confirmed in a 6 -week-old boy following pathological newborn screening. The patient had a stable course of CF under standardized continuous therapy. At the age of 5 years, osteosarcoma of the left proximal humerus was diagnosed without evidence of metastases. Neoadjuvant chemotherapy, including doxorubicin, cisplatin and methotrexate, was administered for 10 weeks. The patient tolerated this therapy relatively well, with a continuous antibiotic prophylaxis of cefuroxime without experiencing major complications; in particular, no pulmonary exacerbations were observed as a consequence of immunosuppression or mucosal toxicity. The tumor responded well, and amputation of the limb was avoided via the use of 'clavicle per humerus' osteosynthesis. Postoperatively, compartmental syndrome occurred, requiring management by fasciotomy. Adjuvant chemotherapy was applied thereafter again, without major toxicity that would have required dose reduction. Under intensive physiotherapy, the mobility of the left arm and hand was deemed to be satisfactory. The coincidence of CF with osteosarcoma is extremely rare, and, to the best of our knowledge, has not been previously described. Under antibiotic prophylaxis, antineoplastic treatment was possible without major complications during neutropenia.
\end{abstract}

\section{Introduction}

Despite great improvements in disease management that have allowed more than half of the patients to reach adulthood, cystic fibrosis (CF) remains a life-threatening condition

Correspondence to: Dr Kenichi V. Okuda, Department of Pediatrics, University Hospital 'Carl Gustav Carus', Technical University, Fetscherstr 74, D-01307 Dresden, Germany

E-mail: okuda@uniklinikum-dresden.de

Key words: osteosarcoma, child, cystic fibrosis, chemotherapy if major complications affect lung and gut function $(1,2)$. Diagnosis of cancer, in addition to $\mathrm{CF}$, is rare, and challenges multidisciplinary treatment algorithms in terms of newly diagnosed patients and those with the pre-existing condition. In children, malignancies are rare, and thus far, no case of treatment of osteosarcoma in a pediatric $\mathrm{CF}$ patient has been described. Osteoblastic osteosarcoma is the most frequently occurring osseous malignancy in children and adolescents. Typically, this tumor type is localized in the lower long bones (75\%), and less frequently in the upper long bones (11\%) (3), and generally there is an improved prognosis in childhood. However, the 6-year overall survival rate has not improved during the last 40 years, remaining at a plateau of $70-80 \%$ (4). Aggressive and long-lasting neoadjuvant and adjuvant antineoplastic chemotherapies are essential, although these treatments are associated with prolonged neutropenia and harbor the risk of bacterial infection (5). The total duration of chemotherapy usually exceeds 6 months on the condition that the tumor response to neoadjuvant treatment was satisfactory. Grade 3-4 toxicity according to the World Health Organization (WHO) common toxicity criteria (6) is quite common: 83,58 , and $27 \%$ of patients experience neutropenia, infectious complications, and mucositis, respectively. The essential local treatment approach in osteosarcoma is total compartment resection, ideally with limb salvage by endoprothesis or autologous bone implantation instead of amputation. Although the survival rate is similar with either approach, an improved function is achievable with limb salvage (7). However, local complications are more common in patients undergoing limb salvage. In the present study, the case of a boy with CF who was successfully treated for osteoblastic osteosarcoma is described.

\section{Case report}

CF was suspected in the Guthrie blood spot screening test from a male newborn, which revealed elevated levels of immunoreactive trypsinogen and pancreatitis-associated protein, followed by a positive sweat test. Diagnosis was confirmed by molecular analysis, unraveling the homozygous carrier status of mutation $\Delta \mathrm{F} 508$. The boy's condition was maintained at an excellent level under a treatment comprising secretolysis using inhalation with hypertonic saline and acetylcysteine, salbutamol and ipratropium bromide, paralelled with 
A

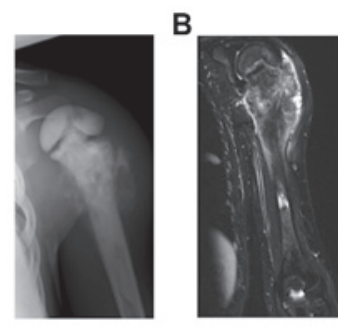

C

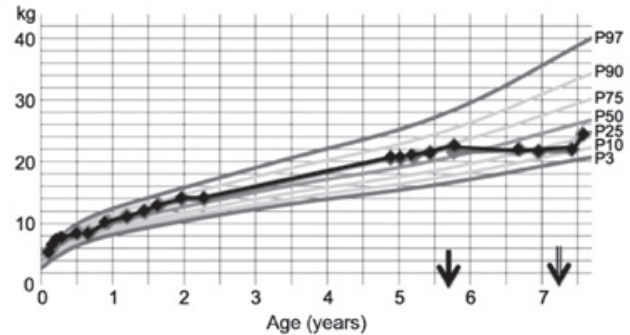

D

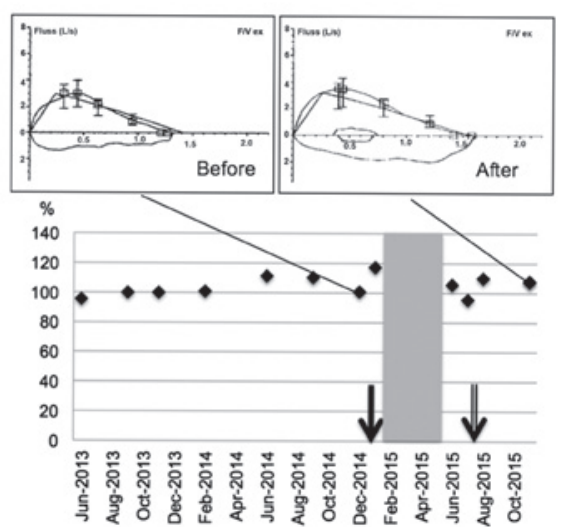

E

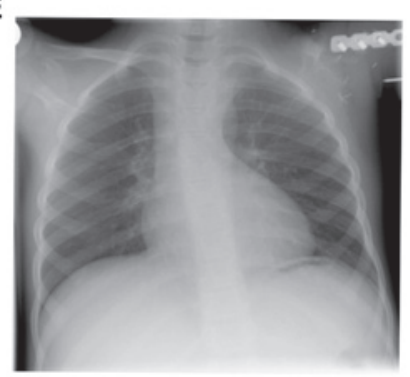

Figure 1. Key findings associated with the course of the disease in a boy with coincidence of CF and osteosarcoma. See the 'Case report' section of the text for further details. (A and B) Conventional imaging procedures, such as (A) an X-ray scan and (B) T2-weighted MRI imaging, revealed the tumor presenting as an $\sim 9 \times 4.8 \mathrm{~cm}$, large, inhomogeneous structure of the left humerus. (C) Course of body weight, showing no gain in weight during the antineoplastic treatment (the start and end of the treatment are denoted by the arrows along the x-axis). (D) Lung function monitored as FEV $_{1}$ (expressed as the percentage predicated of normal controls). Each diamond represents a single measurement performed during the course of CF treatment. The flow volume loops from the time points prior to and following the end of antineoplastic treatment (single arrow: Diagnosis of osteosarcoma; double arrow: End of the antineoplastic chemotherapy) During the first half of the antineoplastic therapy (denoted by the gray shaded area), no spirometry was performed due to immobility associated with the surgical procedures and postsurgical complications. (E) Conventional chest X-ray following the end of the antineoplastic treatment. MRI, magnetic resonance imaging; $\mathrm{FEV}_{1}$, forced expiratory volume in $1 \mathrm{sec}$.

physiotherapy, pancreatic enzyme replacement (PERT) and vitamin replacement therapies, including pancreatic amylases, lipases, proteases, vitamins A, B1, B2, B6, B12, C, D, E, folic acid, biotin, niacin and pantothenic acid. Although the lungs had been permanently colonized with Staphylococcus aureus since the boy was one year old, antibiotic prophylaxis was performed as maintenance therapy for only three years. Regular lung function tests did not reveal any evidence of obstructive or restrictive ventilation disorder (Fig. 1). A chest $\mathrm{X}$-ray revealed no pathological findings. Therefore, hospitalization was never required while the patient presented at the specialized ambulance for $\mathrm{CF}$ in the children's hospital (University Hospital 'Carl Gustav Carus') as required, or every 3 months for a regular follow-up. At the age of 5 years, following a minimal trauma, an indolent swelling of the left shoulder occurred without impaired motility. No further complaints were reported. Conventional X-radiography revealed an irregularly shaped calcified mass at the proximal humerus, suggestive of osteosarcoma. Magnetic resonance imaging (MRI) findings supported this suspicion (Fig. 1). No metastatic disease was detected. Tumor biopsy confirmed a diagnosis of highly malignant osteoblastic osteosarcoma. Prior to treatment, antibiotic prophylaxis with cefuroxime ( $20 \mathrm{mg} / \mathrm{kg}$ body weight per day, twice daily) was initiated to avoid pulmonary infections. Neoadjuvant chemotherapy (the EURAMOS-1 trial) (8) was initiated, featuring the MAP regimen [adriamycin, $75 \mathrm{mg} / \mathrm{m}^{2}$ (A), cisplatin, $120 \mathrm{mg} / \mathrm{m}^{2}$ (P) and methotrexate, $\left.12 \mathrm{~g} / \mathrm{m}^{2}(\mathrm{M})\right]$. Surgery was scheduled following two cycles of MAP, i.e., 10 weeks following the start of the chemotherapy (9). The first febrile episode of unknown origin (FUO) during neutropenia occurred at the ninth week. The patient was treated initially with piperacillin $(200 \mathrm{mg} / \mathrm{kg}$ body weight per day, thrice daily) and tazobactam $(25 \mathrm{mg} / \mathrm{kg}$ body weight per day, thrice daily), and subsequently after $48 \mathrm{~h}$ with meropenem $(60 \mathrm{mg} / \mathrm{kg}$ body weight per day, thrice daily) and teicoplanin (on the first day, $20 \mathrm{mg} / \mathrm{kg}$ body weight per day qd; thereafter $10 \mathrm{mg} / \mathrm{kg}$ body weight per day qd), resulting in defervescence. Neoadjuvant chemotherapy was delayed for one week, and completed thereafter. MRI imaging revealed satisfactory tumor regress, therefore making a limb salvage surgical approach possible. Compartment resection of the proximal humerus was performed, and replacement of the humerus was performed by the left clavicula method (termed the 'clavicula pro humero' method, after Winkelmann) (10). Tumor histology following resection revealed a good response (grade 3, according to Salzer-Kuntschik) (11) to neoadjuvant treatment. The postoperative course was complicated by compartmental syndrome 2 days after surgery. Fasciotomy was performed, followed by a stepwise wound closure. This complication caused a transient deficiency of sensory and motoric function of the left forearm and hand, which were markedly improved following intensive physiotherapy. At 2 weeks postoperatively, the patient experienced 2 days of FUO, which was resolved promptly by antibiotic treatment. As adjuvant treatment, the patient received four cycles of MAP regimen. Up to week 27, the patient tolerated this chemotherapy without 
delay. In week 28, the patient experienced fever again, and antibiotics (piperacillin and tazobactam; dosages as detailed above) were administered for 5 days. Treatment was concluded at week 29 without further complications. The follow-up thus far is 19 months, in ongoing complete remission. The mobility of the elbow and wrist remain satisfactory.

\section{Discussion}

While the occurrence of osteosarcoma at a young age, as for our patient, is very uncommon (it typically occurs at puberty), the coincidence of $\mathrm{CF}$ and malignancy is an extremely rare event in childhood. The worldwide incidence in Caucasians of osteosarcoma is 4.4:1,000,000 at an age of 0-22 years (3) while the incidence of $\mathrm{CF}$ is $1: 2,500$ (12). Therefore, the coincidence of $\mathrm{CF}$ and osteosarcoma can be calculated to be as low as $1.76 \times 10^{-9}$. The case presented here is, to the best of our knowledge, the first case of a pediatric patient with $\mathrm{CF}$ challenged by osteoblastic osteosarcoma. As cancers occur predominantly at an older age, experience of how chemotherapy is tolerated by $\mathrm{CF}$ patients is rather limited. However, colorectal or pancreatic carcinoma represent exceptions that are observed as comorbidity of CF in early adulthood. This coincidence may be attributed to chronic inflammation in CF (13-16), and the frequency increases, particularly following lung transplantation due to immunosuppression. Previous reports $(17,18)$ have emphasized that a high caloric fatty-based food intake, including by means of percutaneous endoscopic gastrostomy, becomes very important in cancer therapy once mucositis hinders oral food intake. In CF patients with malignancies, this represents a major challenge, as the recommended additional energy intake in CF itself compared with healthy controls should be increased by $100 \mathrm{kcal} / \mathrm{kg}$ daily. However, no detailed guidelines exist to date. Under continuous PERT, the patient in the present case report thrived well, until antineoplastic chemotherapy was started (Fig. 1). However, the chemotherapy-associated loss of weight issue was resolved quickly following the end of treatment. Longitudinal growth remained unimpaired. Pulmonary infection during neutropenia also represents a major challenge in oncology. Younger CF patients with only little, or no, pre-damaged lung tissue have an improved initial constitution. Our patient had no permanent airway colonization with Pseudomonas. As the patient's lungs had been colonized intermittently with typical bacteria, such as S. aureus, Haemophilus influenza, Serratia marcescens, and Escherichia coli, since he was 1 year old, cefuroxime was administered during the neutropenic phases. No pulmonary complications were observed, and the patient's lung function remained stable and unchanged during the chemotherapy. The present authors therefore conclude that this prophylaxis worked successfully, although no general recommendations may be deduced from this single case. Immobility following surgery hindered the physiotherapy required daily for respiratory hygiene. In the opinion of the present authors, taking this risk was considered to have been justified, when balancing temporary immobility caused by surgical procedures with avoiding limb amputation. Overall, our patient tolerated treatment for osteosarcoma well, experiencing only two FUO episodes during neutropenia. The present case is intriguing, not only due to the rare coincidence of $\mathrm{CF}$ with osteosarcoma in young childhood, but also since antineoplastic treatment was manageable without exacerbation of CF. In conclusion, it may be speculated that chemotherapy against osteosarcoma may be applied in pediatric patients with $\mathrm{CF}$, with the caveat that defined precautions are followed.

\section{References}

1. Welsh MJ and Smith AE: Molecular mechanisms of CFTR chloride channel dysfunction in cystic fibrosis. Cell 73: 1251-1254, 1993.

2. Dodge JA: A millennial view of cystic fibrosis. Dev Period Med 19: 9-13, 2015.

3. Mirabello L, Troisi RJ and Savage SA: Osteosarcoma incidence and survival rates from 1973 to 2004: Data from the surveillance, epidemiology, and end results program. Cancer 115: 1531-1543, 2009.

4. Bielack SS, Kempf-Bielack B, Delling G, Exner GU, Flege S, Helmke K, Kotz R, Salzer-Kuntschik M, Werner M, Winkelmann W, et al: Prognostic factors in high-grade osteosarcoma of the extremities or trunk: An analysis of 1,702 patients treated on neoadjuvant cooperative osteosarcoma study group protocols. J Clin Oncol 20: 776-790, 2002.

5. Mueller EL, Walkovich KJ, Mody R, Gebremariam A and Davis MM: Hospital discharges for fever and neutropenia in pediatric cancer patients: United States, 2009. BMC Cancer 15: 388, 2015.

6. Trotti A, Byhardt R, Stetz J, Gwede C, Corn B, Fu K, Gunderson L, McCormick B, Morrisintegral M, Rich T, et al: Common toxicity criteria: Version 2.0. an improved reference for grading the acute effects of cancer treatment: Impact on radiotherapy. Int J Radiat Oncol Biol Phys 47: 13-47, 2000.

7. Mavrogenis AF, Abati CN, Romagnoli C and Ruggieri P: Similar survival but better function for patients after limb salvage versus amputation for distal tibia osteosarcoma. Clin Orthop 470: 1735-1748, 2012

8. Whelan JS, Bielack SS, Marina N, Smeland S, Jovic G, Hook JM, Krailo M, Anninga J, Butterfass-Bahloul T, Böhling T, et al: EURAMOS-1, an international randomised study for osteosarcoma: Results from pre-randomisation treatment. Ann Oncol 26: 407-414, 2015.

9. Bielack SS, Smeland S, Whelan JS, Marina N, Jovic G, Hook JM, Krailo MD, Gebhardt M, Pápai Z, Meyer J, et al: Methotrexate, doxorubicin and cisplatin (MAP) plus maintenance pegylated interferon alfa- $2 b$ versus MAP alone in patients with resectable high-grade osteosarcoma and good histologic response to preoperative MAP: First results of the EURAMOS-1 good response randomized controlled trial. J Clin Oncol 33: 2279-2287, 2015.

10. Winkelmann WW: Clavicula pro humero-a new surgical method for malignant tumors of the proximal humerus. Z Orthop Ihre Grenzgeb 130: 197-201, 1992.

11. Salzer-Kuntschik M, Delling G, Beron G and Sigmund R: Morphological grades of regression in osteosarcoma after polychemotherapy - study COSS 80. J Cancer Res Clin Oncol 106 (Suppl): S21-S24, 1983.

12. Scotet V, Duguépéroux I, Saliou P, Rault G, Roussey M, Audrézet MP and Férec C: Evidence for decline in the incidence of cystic fibrosis: A 35-year observational study in Brittany, France. Orphanet J Rare Dis 7: 14, 2012.

13. Demeyer S, De Boeck K, Witters P and Cosaert K: Beyond pancreatic insufficiency and liver disease in cystic fibrosis. Eur J Pediatr 175: 881-894, 2016.

14. Fink AK, Yanik EL, Marshall BC, Wilschanski M, Lynch CF, Austin AA, Copeland G, Safaeian M and Engels EA: Cancer risk among lung transplant recipients with cystic fibrosis. J Cyst Fibros 16: 91-97, 2017

15. Maisonneuve P, Marshall BC and Lowenfels AB: Risk of pancreatic cancer in patients with cystic fibrosis. Gut 56: 1327-1328, 2007.

16. Neglia JP, Wielinski CL and Warwick WJ: Cancer risk among patients with cystic fibrosis. J Pediatr 119: 764-766, 1991.

17. Yen EH, Quinton $H$ and Borowitz D: Better nutritional status in early childhood is associated with improved clinical outcomes and survival in patients with cystic fibrosis. J Pediatr 162: 530-535.e1, 2013.

18. Steinkamp G and von der Hardt H: Improvement of nutritional status and lung function after long-term nocturnal gastrostomy feedings in cystic fibrosis. J Pediatr 124: 244-249, 1994. 